\title{
An Analysis on Dust Avoidance Effect of the Building Complex of Small Xitian in Xi County
}

\author{
Dongdong Yang ${ }^{1}$, Wenqing $\mathrm{Wu}^{2, \text { * }}$ \\ ${ }^{1}$ Institute for History of Science and Technology, Shanxi University, Taiyuan, China \\ ${ }^{2}$ School of Philosophy and Sociology, Shanxi University, Taiyuan, China \\ Email address: \\ 201810502005@email.sxu.edu.cn (Dongdong Yang), wuwq@sxu.edu.cn (Wenqing Wu) \\ ${ }^{*}$ Corresponding author
}

\section{To cite this article:}

Dongdong Yang, Wenqing Wu. An Analysis on Dust Avoidance Effect of the Building Complex of Small Xitian in Xi County. Journal of Civil, Construction and Environmental Engineering. Vol. 5, No. 3, 2020, pp. 52-60. doi: 10.11648/j.jccee.20200503.13

Received: July 2, 2020; Accepted: July 17, 2020; Published: July 28, 2020

\begin{abstract}
The building complex of Small Xitian in Xi County, Shanxi province has a peculiar effect of dust avoidance. As for the unique phenomenon, the previous studies only used the folk legend "Dust Avoidance Beads" to describe the hung sculptures of the Great Hall unilaterally, without systematic and scientific analysis. Through the ancient literature analysis and field measurement, combined with the building climatology and principle of air clean technology research, it shows in this article the western pure land of bliss is an ideological pursuit of the Small Xitian building complex. The relatively closed building site can effectively prevent the invasion of dust carried by the monsoon, and the scattered and well-proportioned building layout has the function of self-regulation and self-output of dust. In addition, the internal structure of the Great Hall has the performance of going against the dust adhesion and the dilution of dust concentration. As one of the representative buildings to avoid dust systematically, Small Xitian complex is integrated into the connotation of ancient architectural art, natural science knowledge and religious culture in China. This can be a supplement to the study of the history of ancient Chinese architecture and provide technical support for the protection of ancient temple architecture.
\end{abstract}

Keywords: Small Xitian, Air Cleaning Technology, Building Site Selection, Architectural Layout

\section{Introduction}

Small Xitian lies in the northwest of Linfen City, Shanxi Province, southeastern part of Lvliang Mountain large anticline axis. It is found that its building complex is not only unique in design and technology, but also has a peculiar dust-proof effect. Especially its Grand Hall is still brilliant, spotless even it has passed more than 300 years. This paper makes a systematic analysis on the location of Small Xitian, the layout of the buildings and the internal structure of Grand Hall to make a scientific explanation of the dust avoidance mechanism of building complex of Small Xitian.

\section{Small Xitian and Pure Land Culture}

The original name of Small Xitian in Xi County is Thousand-Buddha temple. In Qing Dynasty, Kangxi 48 years (1709), XiZhou Annals, Volume 1, Temple View recorded: "For
Thousand-Buddha Temple locating outside of the north gate, then is called Small Xitian." When the temple was repaired during the Republic of China, the name of Thousand-Buddha Temple was replaced by Small Xitian at the horizontal tablet, and the original name was gradually forgotten. The reasons for the changing of name are: Firstly, its meaning echoed virtuality and reality of the Buddhist paradise in the Buddhist Sutra because of the coincidence of geography and Thousand-Buddha temple' facing east, hence getting the forehead title of "Road into the West." Secondly, there was a large-scale opening temple in the southern part of the town at that time, commonly known as the Big Xitian because of monks and laymen's climbing after its completion of the construction. Therefore, it was called "Small Xitian." [1]

According to the records of Emperor Guangxu, Qing dynasty in Shanxi General Annals: Thousand-Buddha Temple, named Small Xitian by scholars, which lied outside the north gate of the State. It was built by Daoliang Shi during the year of Chongzhen, Jiyi lunar year. In the 24th volume of The 
Chronicles of the Xi State by Emperor of Kangxi, it was recorded in the inscription on the tablet Thousand-Buddha Temple by Chengxiang Li: "Beginning from the year of Jiyi, finishing construction until the year of Jiashen."'[2] According to this judgment, the early architecture of Small Xitian was founded during the reign of emperor Chongzhen from the second year to the seventeenth year in Ming dynasty, which lasted 15 years in all. Of the small Xitian's founders, Jichun Yang in Ming Dynasty wrote in the inscription on the tablet Thousand-Buddha Temple: "Master Dongming is a real pioneer." Chengxiang $\mathrm{Li}$ in Qing Dynasty recorded in Monument to Thousand-Buddha Temple: "A monk named Dao Liang from Yan State lives in Xi Mountain. Because the shape of Xi Mountain looks like a phoenix, so it gets the name of Phoenix." Dongming and Daoliang were actually the same person, Dongming was his real name, Daoliang was his alias. ([1] P30-46) Dongming had been a monk in Wutai Mountain since his childhood. For thirty years, he had been practicing meditation, but he was always self-motivated because he supposed he had not been mastered thoroughly what he learned. When he was in his middle age, he went to the south and lived in the Pure Land with Huiyuan from Lushan Mountain, whom was his ancestor teacher. He later became a scholar by understanding and learning gradually. And then he preached and propagandized Buddhism from south to north. On his way to have a lecture, he happened to pass by Xi State, so he made the western paradise miniature in Phoenix Mountain and created a pure and holy temple.

All kinds of buildings in the temple were made by builders who relied on their own cultural quality, creative spirit, coupled with the natural environment of the material conditions. It was also in physical form of the combination of the religious ideas, religious emotion or feeling. Furthermore, it was also a gathering place for those who believed in Buddhism to engage in Buddhist activities, thus affecting and changing the psychology and behavior of those who believed in Buddhism. [3] The purpose of building Pure Land temple is to create a pure land of Buddhism so that people who are troubled by worldly troubles can feel their pure nature in a pure and elegant environment. Through the silent cultivation of environmental landscape, it shows people a way to become a Buddha through returning to the origin and seeing the nature of the heart. [4] Seeking past pure land and gaining of liberation are the urgent desires of the builders of the Small Xitian, and the characteristics of the times when the Pure Land culture flourished. The Small Xitian building Complex not only embodies the connotation of the Pure Land culture and gives people the idea of symbolism, but also has the design system of "external appearance corresponds to inside". The exterior environment and interior structure both convey the essence of the Pure Land culture's pursuit of the purity of the building complex.

In the external environment, the unique natural environment of Small Xitian is a vivid reflection of the pure land of bliss. Chengxiang Li in Qing Dynasty recorded in Monument to Thousand-Buddha Temple: "Out of the north gate of Small Xitian building complex, there are mountains facing around the State which look like phenix's wings, hills on its back, next to a flowing stream. The shape of the mountains is prostrate with the feeling of flying, in the form of a vertical and horizontal vein, a state of flying back." ([1], P94) The building complex is surrounded by mountains on three sides, with steep cliffs of earth on both sides, in front of the temple there is a river surrounded by ancient trees and clear water, and forms a natural landscape. In other words, the landscape of building complex is so wonderful that it just looks like the description of the Pure Land of Bliss in the classic book of Buddha's Interpretation of Amitabha Sutra: "Riputra, the kingdom of Heaven, with seven columns, seven nets, seven trees, and eight rivers of merit, is surrounded by four treasures. Therefore, the name of the land is called Land of Bliss." [5] As Maomei Li of the Ming dynasty wrote in $A$ Journey to "Thousand-Buddha Temple: "After a few more visits to this place, some of the earthly distractions have been disappeared, and people are more than willing to give up everything possessed to follow the knowledgeable sages ([1], P152) At the same time, it also follows and conforms to Chinese mountain temple site tradition of leaning on the shade side and facing the sun side, its back leaning on the mountain and its front facing the river to retain good Chinese Feng Shui.

In terms of the internal structure, the Small Xitian building complex not only maintains the continuation of the ecological and cultural environment, but also innovates the architectural structure. In the overall organizational concept of the building complex, the design idea of introduction and brief formal language show the pursuit of the cultural connotation of Pure Land of Buddhist. [6] From the entrance to the gate and the main building, its rational and ingenious layout in the limited topographic environment, turns the hearts of believers from "vulgar soil" to "pure land". Of functional types, the Small Xitian building complex is naturally divided into three parts: Front Courtyard, Lower Courtyard and Upper Courtyard due to the shape of the mountains. The Front Courtyard has a broad vision, offering people a feeling that everything under the mountain is included among the vastness of Buddhism. The Lower Courtyard is so closed, which has the effect of convergence vision and mood; the Buddhist sculptures in the Upper Courtyard were lifelike, making people feel as if they were in the Pure Land World of Bliss. The overall architecture meets practical and spiritual functions. In terms of organizational structure, Small Xitian uses the combination design of single body building to set Great Hall of the Pure Land of Bliss in a place with obvious height difference, to achieve the purpose of absolute domination. It conforms to the internal language logic mode of "the sage still exists despite of the six harmonies" in the space standard of ancient buildings in China.

\section{Dust-Avoidance Effect of Small Xitian Complex Site}

The landform of Xi county is high in the northeast and low in the southwest, and most of the landform is between 950 
meters and 1300 meters above sea level. The highest peak of Zijin Mountain in the east is 2012 meters above sea level, which is the highest peak of lvliang Mountain in the southwest of Shanxi Province. The lowest elevation of Hucheng village in the south is 760 meters, and the relative height difference is 1252 meters. There are many ravines in Xi county. Between the mountains, there are two rivers, seven tablelands and eight gullies surrounded. There are 1124 gullies with a length of more than 1 kilometers and the gully density of 3.4 kilometers per square kilometer, presenting strip geomorphologic features (Figure 1). Studies of climate architecture show that the topography, humidity, and vegetation of a region all influence the pollution of the building surface. [7]

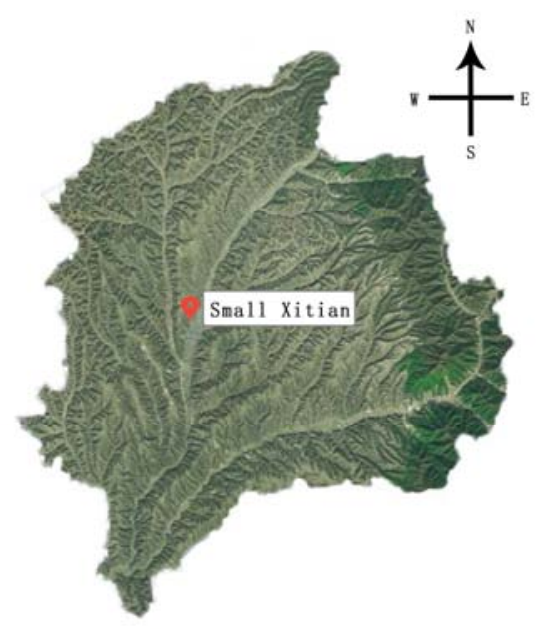

Figure 1. Topographic map of Xi county.

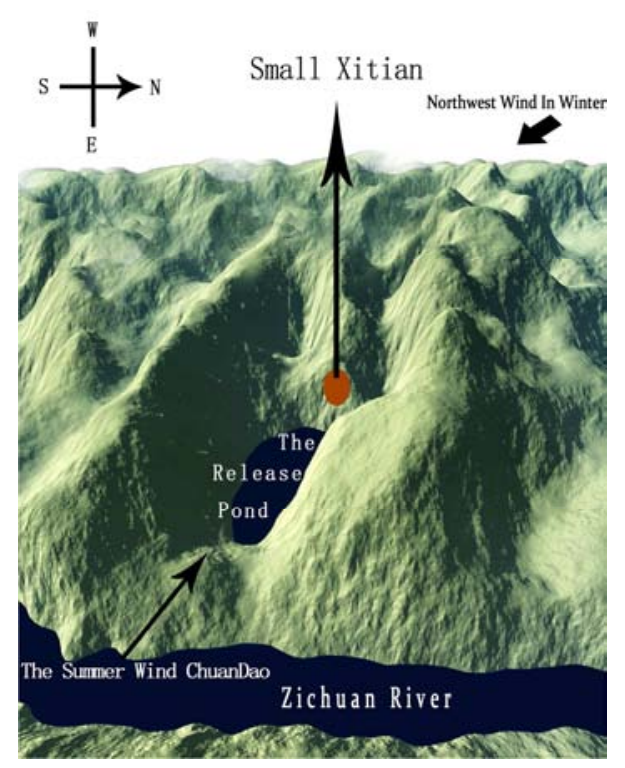

Figure 2. Terrain analysis map of Small Xitian.

In terms of local terrain, Small Xitian is located on the top of the Phoenix Mountain. It is not a solitary peak, but it is in the north-south direction instead, belonging to one of the branches with west slope of the plateau in the east-west direction, and Phenix Mountain intersects with it in a T-shape. Its altitude is about 1025 meters above sea level. The top of the mountain is about 100 -meter long and 10 meters to 40 meters wide, showing a sharp-mouth shape. The mountains on both sides of the north and south are about 100 meters higher than Phenix Mountain, which is encircling, rushing through Zichuan river, shaped like a phoenix with wings, and relatively closed terrain. Although the wind often blows, but the wind is difficult to come, and the dust rarely invades. The north and south sides of the Small Xitian complex are steep slopes with brownish red clay, sub clay and sand pebble layers on the geological landform. The soil layer is of good viscosity and less dust in windy weather. The slope of the north is 1:0.6, the vertical height is 40 meters, the depth of the ditch is 300 meters; the slope of the south is $1: 1$, the vertical height is 25 meters, and the depth of the ditch is 50 meters. (Figure 2) Because the hillsides and valley are heated unevenly, the valley wind is easy to form during the day and the mountain wind is easy to form at night. The valley wind accelerates the air flow on the north and south sides of the small Xitian building complex and dredges the dust of the surrounding environment of the building complex. [8]

According to the research on the influence of environmental humidity on dust removal performance, the force between dust particles is an important factor affecting dust accumulation. In addition, increasing environmental humidity can increase the force between dust particles of different particle sizes. [9] In Ming Dynasty, Jichun Yang recorded in the Inscription on the Tablet of Thousand-Buddha Temple: "There is a mountain in the northwest corner of Xiangcheng shaped like a phoenix facing southeast. The middle part of the temple is like its body, its southern and northern rocks are like spreading wings, and both sides of the flanks are the small streams. Many of the tributaries flow down from the north end into the Yellow River. Some of the streams run west to the sea where the waters gather to the south. The State of Zhou is the only place where the river flows out. So called Xunsheng district."([1], P94) Nowadays, springs still flow out from the valleys all the year round. In the valley, a relatively wide S-shaped water storage project is built-- the Release Pond; The distance between the east and the Zichuan river is less than 300 meters. A herringbone reservoir is built to the east. According to the actual measurement by the author, the absolute humidity of the surroundings in Small Xitian is $35.6 \%$, higher than that in other areas of $\mathrm{Xi}$ county in the absence of rain. The higher the humidity is, the higher the air pressure of water vapor in the air is, the water vapor is more likely to condense between the dust particles, which increases the adhesion between the dust particles. The friction resistance and gravity change of the dust particles limit the relative slip between the dust particles, and the natural landing is more significant, which effectively prevents the external dust from entering the interior of the Small Xitian building complex.

Plant purification is an important dust prevention measure. Kuang Song in Qing Dynasty wrote in the Record of Rebuilding Thousand-Buddha Temple: "There are secluded gullies on both sides with several kinds of ancient trees. It is a place of happy meditation for monks and hermits. Taking a walk on the bridge above the stream in leisure time, it will be 
seen the trees growing robustly and the springs flowing quietly." Fuhai Jin of the Republic of China wrote in Jinqiao Monument of Small Xitian: "Once there were small saplings all around it, but now the temple takes on a new look, and the trees are so green and verdant that they are regarded as the crown of scenic spots in the county." ([1], P94-96) Today, Small Xitian is surrounded by thick trees in the valley which almost cover the sky. Its total coverage of plants is more than $70 \%$. Good vegetation around it played a good role in dust retention. According to the research on the principle of air cleaning technology, there are obvious differences in the amount of dust retention among different tree species. The roughness of leaf surface, stomatal density, opening size, villi density and distribution characteristics all affect the dust retention ability of leaves. [10] The gully wetlands on the north and south sides of Small Xitian are mixed forests of needles and broad leaves, mainly cypress, elm, poplar and willow. The vegetation on the hillside is mainly mixed with coniferous and broad-leaved shrub grass, and it is mainly dominated by pine, cypress, toon and elm. The other areas are mixed forest of shrubs and broad leaves with rich herbaceous plants etc. There are more elms, sophora and toon at Small Xitian. Their leaves are broad, flat and stiff, with coarse, hairy and large total leaves, so they are more efficient in dust retention and dust reduction. [11]

\section{Dust-Avoidance Effect of the Layout of the Small Xitian Building Complex}

The overall building area of Small Xitian is only more than 1,100 square meters. In the extremely limited space, there are more than 20 rooms, and a hole is used as the door to separate and connect the three courtyards of the Front Courtyard, the Lower Courtyard, and the Upper Courtyard. Although the overall layout is guided by the traditional building axis and takes the form of left and right symmetrical, it does not flow in stylization or stick to the stylization, but adopts a variety of techniques, such as turning back and forth, staggering, sometimes opening, or sometimes claustrophobic etc to endow the Small Xitian building complex with the function of avoiding dust. (Figure 3)

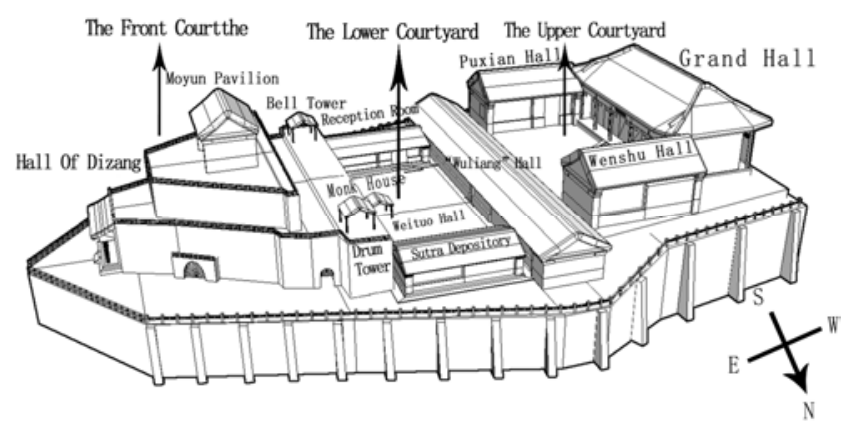

Figure 3. 3D panorama of Small Xitian building complex.

Jichun Yang of the Ming dynasty wrote in the Inscription on the Tablet of Thousand-Buddha Temple: "Obtaining a quaint and peaceful trail by digging through a mountain... then create three brick-arched structures. The front of the temple is adorned with doors, windows and halls, both sides of the temple are used as side rooms. Opposite is the Weituo Temple, followed by the houses of three living monks. There are doors on either side in order to reach the Front Courtyard, there is also a secluded trail in the forecourt, named "Xiaodongtian", which leads to the Moyun pavilion...A total of five temples were built on top of the three brick-arched structures." Wuliang Hall which located in Small Xitian's Lower Courtyard, two unknown rooms facing the principle rooms; Weituo Hall and the Moyun pavilion on Gutong peak in the Front Courtyard had been built in the initial period of creation, "Floor Hall" at the Upper Courtyard named Main Hall were also preliminary completed. Qingya Liu of Qing Dynasty Recorded in a Brief Introduction to the New Two Corridors of Wenshu and Puxian: "Master Dongming pioneers the shape of the Upper Court... Five halls were built, but before the two sides of the halls were finished, Dongming went to the western paradise. His disciple's built three auxiliary temples on each side of Wenshu and Puxian hall to provide support for the five great halls." The two Chambers of the Upper Courtyard, named Wenshu and Puxian, were built within the space layout planning of the Thousand-Buddha temple founded by Master Dongming, and later his disciples completed the final construction. [12] (Figure 4) Although it has been supplemented and renovated, the function of dust avoidance of the overall architectural pattern at Small Xitian has not changed.

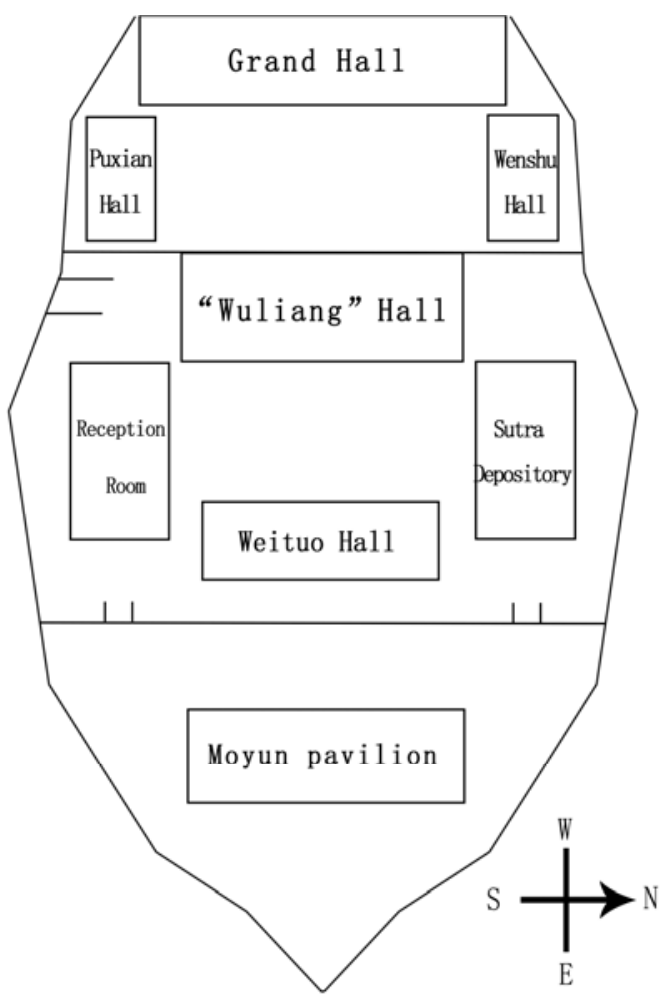

The layout plan of the Hall at the beginning. 


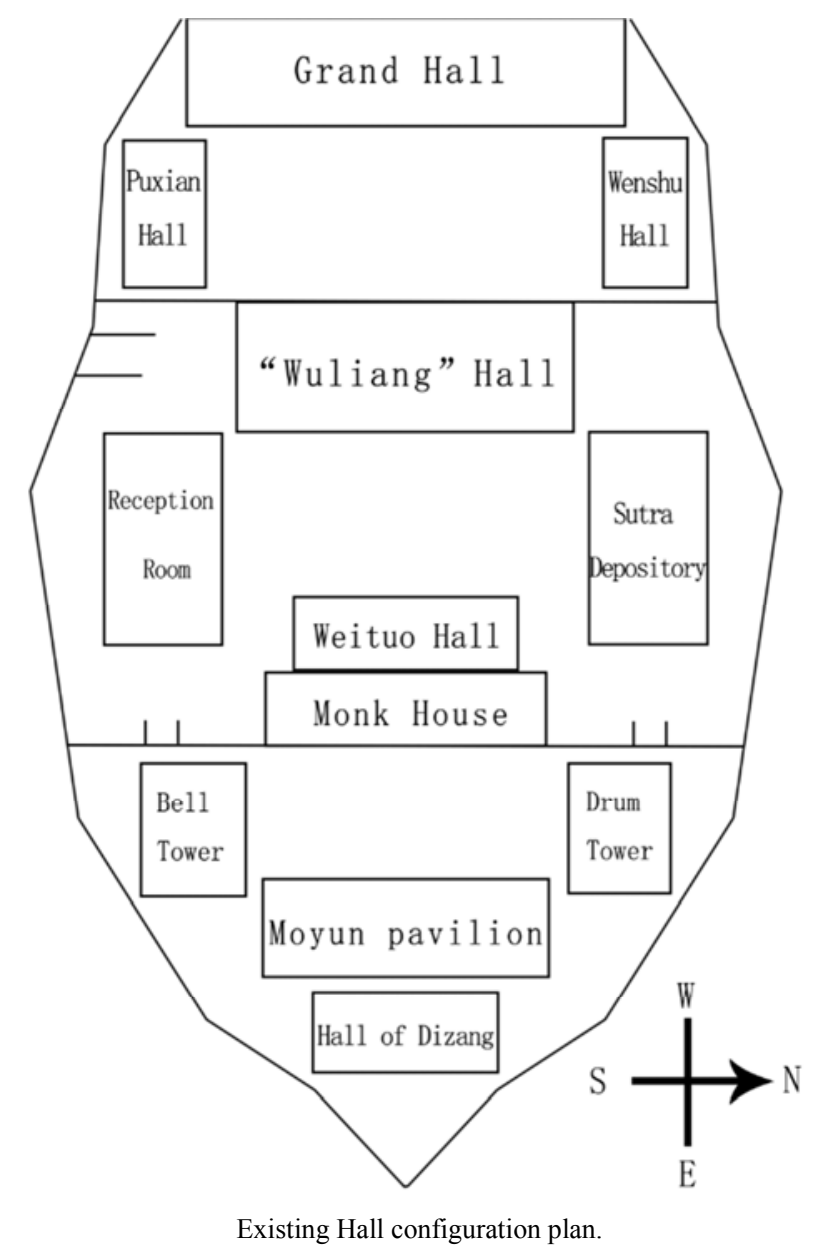

Figure 4. The plane figure of Small Xitian complex.

\subsection{The Building's Clog Ratio and Curved Mountain Gate}

From the perspective of modern architectural theory, diversified spaces are designed in the planning of Small Xitian building complex, which makes the building become a living organism with the function of automatically adjusting and restoring stable air quality. The main source of pollution wind in Small Xitian comes from the eastern exposure, and the direction of the building complex is basically parallel to the dominant wind. When the buildings are in parallel with the dominant wind, the main parameters affecting air circulation are the width (L) of the unobstructed object beside the building and the height $(\mathrm{H})$ and width $(\mathrm{W})$ of the front facade of the upwind building. Block ratio $\left(\mathrm{R}_{\mathrm{b}}\right)$ is defined as

$$
\mathrm{R}_{\mathrm{b}}=(\mathrm{W} \times \mathrm{H}) /(\mathrm{W}+\mathrm{L})^{2}
$$

The maximum width of Small Xitian building complex is 40 meters, the highest building is about 15 meters, 140 meters away from the north side of the mountain beam, 110 meters away from the opposite side, the blocking ratio of $\mathrm{Rb}$ is less than 0.01, far lower than the normal building layout blocking ratio. ([7], P. 192). According to the inverse relationship between air flow and blocking ratio, the air circulation performance around Small Xitian building complex is very good, the wind flows along the valley on both sides, the air flow over the buildings is easy to cause regeneration of air flow in the lower building space, and the ability of building self-regulating air quality is strengthened.

The first mountain gate in Small Xitian is a cave gate carved out of earth on the mountainside. It is 2 meters high, 1.2 meters wide, and the passage is 20 meters deep. The plane is $\mathrm{S}$-shaped and gradually rising. When the polluted air stream passes through the passage, the dust with large particle mass cannot follow the air stream around the bend due to their inertia, so it breaks away from the flow line and gets close to the wall, which is deposited when they collide with the wall. (Figure 5)

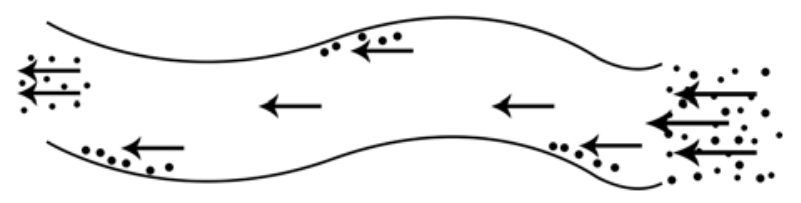

Figure 5. Schematic diagram of dust resistance in the curve.

\subsection{Design of Front Courtyard Vents}

The portals named Yiwulu and Bieyoutian on both sides at the Front Courtyard connect to the Lower Courtyard, are also the main vents inside the building. According to the modern architectural climatology theory, the optimal vent should be at an angle between $30^{\circ}$ to $60^{\circ}$ and the dominant wind direction should be about $60^{\circ}$. The incident angle is bigger, which avoids the generation of wind shadow areas and hinders the ventilation of the yard to the Lower Courtyard. ([7], P192) In the aspect of preventing dust, the outer door of Yiwulu on the north side is 1.7 meters high, 0.9 meters wide, 1 meter deep, and the turning depth is 2.8 meters. The inner door is 1.7 meters high and 0.5 meters wide. The outer door of Bieyoutian on the southern side is 2.5 meters high, 1.3 meters wide and 1 meter deep. The turning depth is 2.9 meters, the internal door is 1.8 meters high and 1 meter wide. This kind of door can only be used in burial buildings, which is very rare in the temple buildings. According to the mechanism of clean air filtration, the plane is shaped like an L and the outside is wide and the inside is narrow, similar to the design of high efficiency filter to block the air passage of separated particles. The head-on resistance effectively intercepts and captures the dust in the airflow. (Figure 6)

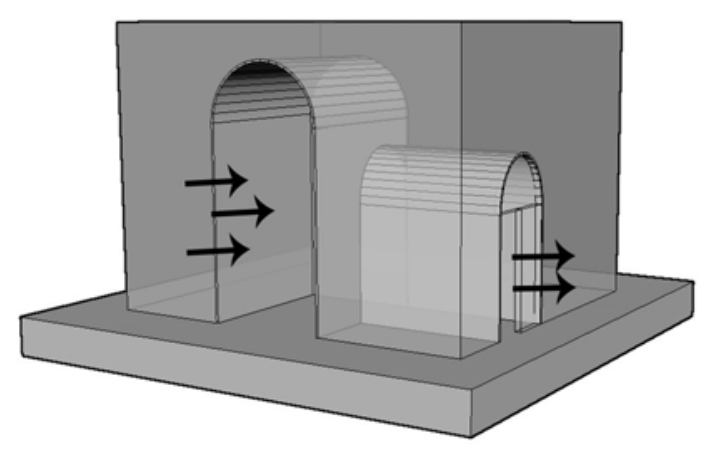

The door of Yiwulu. 


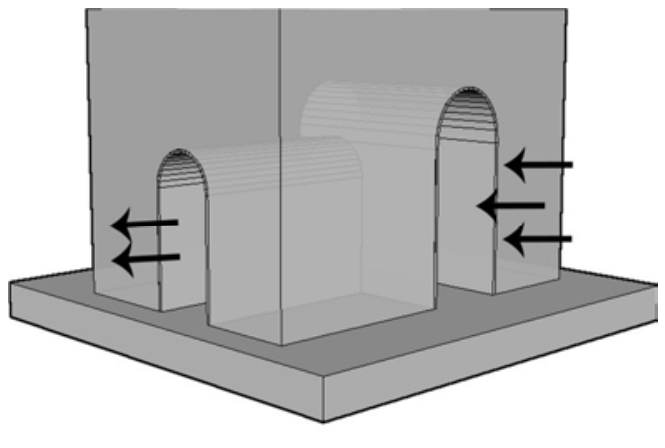

The door of Bieyoutian.

Figure 6. 3D View of the Cave Door on Both Sides of the Front Courtyard.
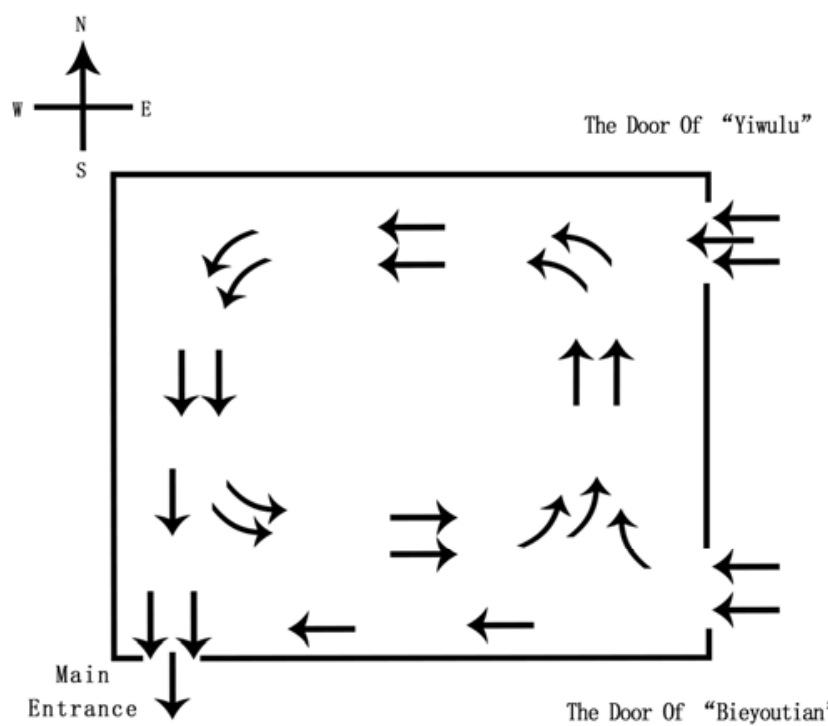

Figure 7. Schematic of the Lower Courtyard vortex.

\subsection{The State of Motion of the Lower Courtyard of Air}

The Lower Courtyard is quadrangle. Wuliang Hall is in the west, Weituo Hall and the Abbot's room are in the east, the Sutra depository is in the north, and the Reception room is in the south. All these rooms form a closed courtyard. According to the principle of fluid mechanics, the airflow in the Lower Courtyard is extremely unstable due to the ventilation of fluid particle both sides of the Front Courtyard. In relative motion, shear stress is generated between layers due to viscosity. Due to the decrease of the section, the flow rate of the northern side of the gate increases, and the shear stress is downstream, while the southern side of the gate is counter-current, forming a vortex body of airflow. Since the flow layer with a higher velocity is in the same direction as the vortex body, and the flow layer with a lower velocity is in the opposite direction of the vortex body's rotation, it will increase the flow rate in the upper troposphere and decrease the flow rate in the lower troposphere. When the velocity of flow increases, the pressure decreases, and the pressure difference is formed perpendicular to the flow direction. When the pressure difference is sufficient to overcome the resistance, the vortex body is pushed out of the primary stream and into the adjacent stratosphere. The front door of Small Xitian acts as a neighbor layer, and the Lower Courtyard has two conditions of vortex formation in the flow layer and rushing into the neighbor layer, which can quickly and effectively eliminate dust pollution caused by incense burning and leading wind. ([11], P267-270) (Figure 7)

\subsection{The State of Motion of Air Currents in the Upper Courtyard}

The Upper Courtyard is also a square courtyard, the difference is that there are only three sides of the aggregation, Grand Hall in the west, Wenshu Hall in the north and Puxian Hall in the south form a relatively closed wind shadow area environment. It is free from external air dust pollution. [13] According to modern architectural climatology, when the building is a parallel arrangement in determinant, there must be a large building spacing in the direction of wind flow. A larger space and a lower building height between the front and back of the building can minimize the loss of wind. The required interval is large, generally required to reach 4-5 times the height of the previous buildings. Those buildings in the Front Courtyard of Small Xitian are about 15 meters high, 5 meters higher than the Main Hall of the Upper Courtyard. The building space is about 14 meters, less than one time of it. At the same time, there are many pines and cypresses in the Lower Courtyard, which make the wind turn upward and cross over the buildings, creating a closed space that can isolate the upper Courtyard in all seasons. (Figure 8)

On the right side of the Wuliang Hall in the Lower Courtyard is a passage to the Upper Courtyard. This passage is a circular cave door that can only accommodate one person. The exit of the passage does not directly lead to the Upper Courtyard, but it winds its way to the Wenshu Hall in the Upper Courtyard. Such a design greatly limits the dust from Lower Courtyard into the Upper Courtyard. (Figure 9) As long as incense is burned in the incense burner in front of the Hall, the resulting dust pollution will always float upward. According to the principle of air cleanliness, due to the continuity of air flow and the mutual supplement between fluids, as well as the relatively closed space environment of the Upper Courtyard, the air flow produces vortex body exchange, which makes the air flow state basically vertical and upward, and automatically diffuses the carrying dust into the fast flowing air flow in the upper layer. ([11], P269)

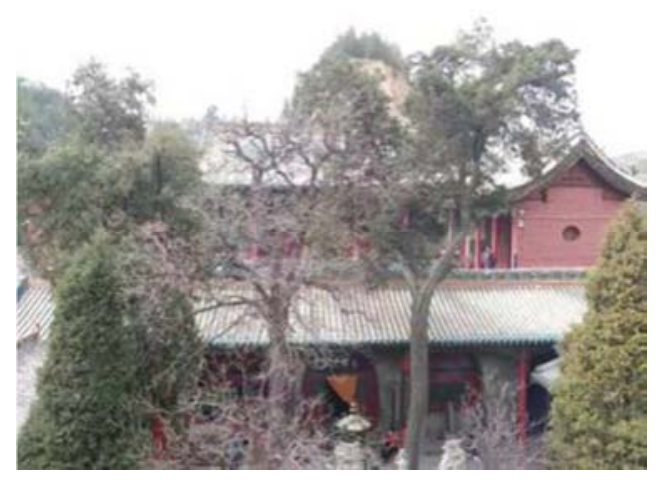

Figure 8. Vegetation of Lower Courtyard. 


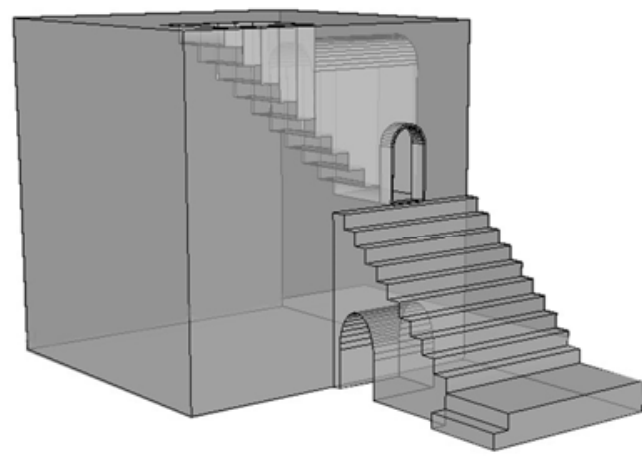

Figure 9. 3D view of access to the Upper Courtyard.

\section{Dust-Avoidance Effect of the Inner Structure of Great Hall}

Great Hall is the core building of both the Upper Courtyard and the Small Xitian. Great Hall has a width of 5 rooms, a depth of 6 rafters and below the front eaves is the hall. The total area is 169.6 square meters. About the clean phenomenon in the hall, it is considered that the Buddha body contains dust-proof beads. Some scholars believe that the hanging figures above the Buddhist Pavilion are inclined forward according to the requirements of the scene. They form a certain angle with the vertical direction of the falling dust to avoid the direct fall of dust. The chance of dust contamination on the front is left to the back of the hanging statue. In fact, the cause of the cleanliness of Great Hall is more complicated.

\subsection{Air Flow in Great Hall}

According to the function principle of one-way flow clean room, to obtain the lower dust content of the whole room, it is required that the dust concentration of the air supply outlet is low, and its dilution function can be fully exerted after entering the room.([11], p279). The only air outlet in Great Hall is the main entrance of it, which is about 1.1 meters wide and 3.1 meters high. As the front of the hall is a shadow area of wind, it is mainly affected by the rise and diffusion of airflow of vortex body from the Lower Courtyard. The dust content in the airflow is low, the air supply speed is low, and the diffusion surface of airflow into the hall is larger, so it plays a mixing dilution effect, in line with the one-way clean room characteristics.

The inner length of Great hall is about 14.2 meters, and the height is about 7.3 meters. The air of entering the hall has a large and parallel winding path. The inner layer does not exchange fluid particles in a short time. With the change of time, the external airflow fills the horizontal section of the whole hall, and the flow layer has fluctuated perpendicular to the direction of fluid movement. The interior of the temple, in the form of panoramic and three-dimensional art, shapes large numbers of characters, plots and scenes in different time and space. In general, the hall is divided into five parts based on five Buddhist shrines. And the various parts are connected into a whole through the ring of rocks, the interlacing of trees, the echo of rooms and the convergence of auspicious clouds.
Before and after the shrine, with a number of columns to form a vertical load-bearing network, through clever hollowed-out connection, a large number of halls and pavilions and hanging figures are constructed which covers most of the Hall's space. In the front and back of the shrine, there are several columns forming a vertical load-bearing network, which connects by artful pierced connection, and sculpts numerous halls, pavilions and hanging figures, to cover most of the space of the main hall in a dense and subtly manner. In the movement of the upper and lower airflow, mainly due to the fluctuation of the temple and the statues of collision, reflux phenomenon is likely to occur on the flow layer on a smaller scale, whirlpool changes between the statues, so that the dust on the statues are blown away, and the original higher concentration of dust in the hall air dilutes. [14] At the same time, due to the static pressure difference between the upper and lower airflow, the diluted airflow forms an air piston, along this cylinder inside of the hall, pushing downward, the air with high dust concentration is squeezed out from the air inlet in order to purify the air. the function of purifying the air is achieved by extruding the air with high dust concentration from the inlet outlet.

\subsection{Indoor Humidity Analysis}

The annual relative humidity in Great Hall is about $50 \%$, which the humidity is relatively high. The water vapor in it is easy to condense between dust particles, which greatly increases the force between dust particles and limits the relative slip between dust particles. At the same time, the influence of environmental humidity on the interaction between dust particles is related to the surface hydrophobicity of dust particles. That is, the stronger the surface hydrophilicity of dust particles is, the stronger the influence of environmental humidity on the force between particles will be. [15] In the case of insoluble condensation nuclei, the condensation of water vapor on it depends mainly on the surface saturation:

$$
\frac{E_{r m}-E}{E}
$$

(Erm is the saturated water pressure on the droplet, and E is the saturated water pressure on the air).

The larger the condensation core is, the smaller the surface saturation required for condensation is. That is, supposed $\mathrm{E}$ is maximum, the smaller the relative humidity of the air is accordingly. On the contrary, the larger the relative humidity is, the larger the moisture absorption of smaller condensation nuclei will be. ([11], P84) From the chemical composition of the dust particles of air inside the hall, it is mainly composed of incense ashes particles by the smaller particle size and easy wet by water. It is an insoluble condensation nucleus with low surface saturation. As a soluble condensation nuclei, the surface of a smaller saturation is advantageous to the formation of a thicker water film on the surface of dust particles by water vapor, increasing the mutual adhesion between dust particles, adding the contact area between airflow and the surface of dust particles, enlarging the 
frictional resistance and gravity, and making the natural fall of dust more significant. [16]

\subsection{Hanging Statue Analysis}

During the Ming and Qing dynasties, the production of colored statue had been very advanced. The internal wooden skeleton enhanced the freedom of the production of colored statue, and it was produced based on this change. Hanging sculpture makes temple art more expressive, which not only gives people a new sense of vision, but also offers more suitable expression of religious significance. Hanging statue at Small Xitian is one of the most representative color sculptures in existence, and is known as the hanging statue masterpiece in the history of Chinese statue art. [17] From the analysis of the surface cleanliness of hanging statue, its inclined structure and painted gold made it have anti-dust effect.

The hanging statue at Small Xitian adopts the way of nearness reduction in perspective, that is, it is intended to reduce the part of the person who is away from the eyes, and gradually expands from the bottom to the top, and tilts forward 3-8 degrees to shape the figure at the top into a state of overlooking. [18] The dust particles entering the hall float in the parallel movement of the airflow. It is generally believed that the particles are on the flat vertical surface, and the inertial settlement can be completely ignored, only the diffusion deposition exists. Under the condition of the same time and the same dust concentration, the amount of dust deposition depends on the contact area between the dust particles and the object surface. The larger the contact area is, the stronger the adhesion will be. The spherical incense ash particles are effective in contact with the hanging statue surface at a single point, decreasing the effective contact area and correspondingly reducing the adhesion. [19] The amount of dust on the surface of hanging statues is less than that on the surface of non-tilted colored statues. (Figure 10)

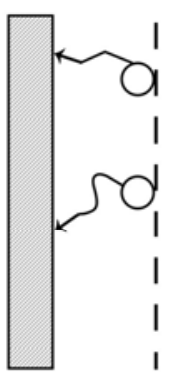

Non-Inclined plane.

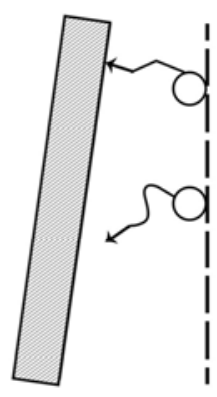

Inclined plane.
Figure 10. Schematic diagram of dust accumulation without inclined plane and inclined plane.

At the same time, the amount of dust particles adsorbed on the object surface is affected by the roughness of the object surface. Improving the surface finish of the object can reduce the attachment of particles. [20] According to Rui Wu's early investigation of the Hall and the analysis of inscriptions in it, during the Ming and Qing dynasties, there was an active construction team with excellent skills in architecture, sculpture and painting. The statues in the hall which took about five or six years to shape, had been repeatedly polished and repaired before adding color, so the clay itself had been particularly smooth. [21] From the aspect of using color, with drawing from the use of traditional painting in the application of color skills, the hanging statues works are imposed a strong color by using a large amount of leachate powder to coat the surface with gold. According to the manager of Small Xitian, the spotlight temperature is high because of taking video to advocate some of the statues are carbonized and become blackish and expose to mud. In addition, the hanging statues are covered with thin and smooth gold, which is not conducive to the adhesion of dust.

\section{Conclusion}

Small Xitian building complex in $\mathrm{Xi}$ county is a typical dust-proof building of ancient temples in China. After investigation and analysis, Small Xitian building complex in $\mathrm{Xi}$ county is a physical carrier created by the pure land world of Buddhism, which is free of dust, filth and evil. Its dust avoidance effect vividly reflects the systematic engineering of building temples to avoid dust in ancient China. In the selection of the site to avoid dust, the effect of terrain, humidity and vegetation on reducing the concentration of dust in the air has been fully considered. When considering the layout of the building complex to avoid dust, people always take advantage of the high and low strewn at random and the zigzags of the loop, so that the air quality of the building complex itself can be self-regulated and the ability to intercept and capture external dust can be strengthened. In the aspect of dust avoidance of single building internal structure, the air flow, humidity inside the building and the hanging sculpture itself ensure the lasting clean phenomenon. The systematic dust avoidance effect of Small Xitian building complex in $\mathrm{Xi}$ county is rare in the history of science and technology at home and abroad, which confirms the scientific nature of the dust avoidance effect of ancient temple buildings in China and plays a reference role in the research on dust avoidance protection of other ancient buildings in China.

\section{References}

[1] Wang, Z. (2004) Small Xitian Annals [M]. Shanxi People's Publishing House, (10) 30.

[2] Cultural and historical data research committee of the committee of the Chinese People's Political Consultative Conference. (1992) Xi county cultural and historical data [M] Printing by Xi County Printing Plant (8): 94.

[3] Li L., Li J., and Ji K. (2017) Research on the concept of harmony in ancient Chinese buildings [M]. Beijing: China Social Sciences Press, (01): 182.

[4] Lv, j., and Zhang, Q. (2011) Design considerations of contemporary Chinese Buddhist temple architecture -- A case study of Zhongtaizen temple and Fafeng Mountain in Taiwan province [J]. Huazhong Architecture, 29 (09): 45-48. 
[5] Jing, Y. (2014) The three Sutras of the pure land [M]. Citic Press, (02). 28.

[6] Chen C. (2018) Chen Congzhou said ancient architecture (Volume 2) [M]. Beijing: Social Sciences Academic Press,(11): 60.

[7] Yang, L. (2010) Building climatology [M], China Building Industry Press (6): 16.

[8] Yan Sh., Zeng Ch., and Wang X. (2020) Numerical Simulation of multi-mountain wind field flow [J]. Thermal Power Generation, 49 (06): 77-83.

[9] Li, N. (2016) Study on the influence of environmental humidity on dust removal performance of different properties, [D]. Beijing University of Chemical Technology.

[10] Sun, Y., Chen, Q., Li, Y., and Yang, S. (2019) Study on the relationship between leaf surface microstructure and dust retention capacity of six green tree species in Kunming city [J]. Journal of southwest Forestry University (Natural Science) 39 (03): 78-85.

[11] Xu, Z. (2014) Principles of air cleaning technology. BJ: Science Press 90.

[12] Sun, Z. (2006) Pure Land and Nirvana [D]. Central Academy of Fine Arts.

[13] Shen G., Weng W., and Wang Y.(2020) Three-dimensional wind field characteristics of a complex mountain [J]. Vibration and Impact, 39 (04): 75-80.
[14] Wang X., Zhou D., and Li Y. (2017) Numerical Simulation analysis of Wind Pressure and Wind Field of Chinese Ancient Buildings on The Peak of Double Eaves [J]. Journal of Shanghai Jiao Tong University, 51 (11): 1287-1296.

[15] Wang Zh. (2019) Study on the influence of microbial concentration level and related parameters in different functional buildings [D]. Beijing University of Civil Engineering and Architecture.

[16] Chen Y., Gu M., and Chen S. (2020) Dispersion law and distribution characteristics of high-drop dust [J]. Journal of Process Engineering, 20 (07): 1-10.

[17] Jiang Zh., Xu Y., and Liu Ch. (2018) Map of Shanxi Ancient Architecture (Volume 1) [M]. Beijing: Tsinghua University Press (08): 243.

[18] Zhang Y. (2018) Hung sculptures analyses [J]. Identification and Appreciation of Cultural Relics, (19): 30-33.

[19] Li, Y. (2007) Study on adhesion mechanics of microparticles on surface and its visualization application [D]. Central South University.

[20] Liu M., Zhang Y., and Guo F. (2019) The influence of surface roughness on the floatability of slime [J]. Coal Science and Technology, 47 (10): 253-258.

[21] Wu, R. (1998) A new exploration of the construction and color sculpture of Grand Hall of Thousand-Buddha Temple, Xi County, Shanxi Province [J]. Cultural Relics, (05): 65-69+99. 